\title{
Pansharpening via Locality-Constrained Sparse Representation
}

\author{
Songze Tang ${ }^{1}$ \\ ts198708@163.com \\ Nan Zhou ${ }^{1}$ \\ nudge@163.com \\ Liang Xiao ${ }^{2}$ \\ xiaoliang@mail.njust.edu.cn
}
${ }^{1}$ Department of Criminal Science and Technology, Nanjing Forest Police College, Nanjing, China
${ }^{2}$ Department of Computer Science and Engineering, Nanjing University of Science and Technology, Nanjing, China

\begin{abstract}
Recently, sparse representation based approaches have been shown an effective performance for pansharpening. However, these methods imposed $\ell_{0}$ or $\ell_{1}$-norm constraints on the sparse coefficients. The local similarity of sparse coefficients was ignored. Motivated by the importance of data locality, in this paper, we propose a locality-constrained sparse representation algorithm for pansharpening, which keeps the data locality during the sparse representation process. The learned dictionary is able to preserve local data structure, resulting in improved data representation. During the sparse coding stage, analytical solutions are provided based on the basis of mathematic deduction. The pansharpening results show that the proposed method is competitive to the other well-known fusion methods.
\end{abstract}

\section{Introduction}

Due to the launch cost and technical limitations, most spaceborne imagery, such as IKONOS, QuickBird, GeoEye, WorldView-2, GaoFen-1 and GaoFen-2 provided separated but complementary product types, i.e., a high spatial resolution (HR) panchromatic (PAN) image and a low spatial resolution (LR) multispectral (MS) image with several spectral bands [1-3]. However, a single PAN image or MS image cannot provide complete descriptions about the scanned region, and incomplete descriptions may influence further applications. Hence, high quality image with simultaneous high spatial and high spectral resolution is urgent. Pansharpening techniques have been developed to produce high quality image by combining the HR PAN and LR MS images [2]. Consequently, it has been the focus of spectral imaging techniques, and many methods have been proposed in the last decades. 


\subsection{Related Work}

Up to now, numerous approaches have been developed. Broadly, three methodologies have been commonly used, namely, the component substitution, the multi-resolution analysis (MRA), and the model-based method [3].

The basic framework of component substitution methods is to transform the MS image into other space using a suitable transformation, and then one component of the MS image in the transformation domain is replaced by the PAN image. The classical component substitution methods are the intensity-hue-saturation (IHS)[4], and principal component substitution (PCS) [5]. Although these methods preserve spatial information accurately, spectral distortion is also generated. The reason is that PAN and MS components are acquired in spectral ranges that overlap only partially. Some variant improvements to the original IHS-based method have been proposed. Rahmani et al. proposed an adaptive IHS method (AIHS) [6], which tried to represent the intensity component by an adaptive linear combination of the MS bands with the combination coefficients obtained by solving an optimization problem. Rasoul and Peyman proposed a new IHS (NIHS) method using texture analysis and genetic algorithm adaption [7].

The MRA methods are based on the injection of spatial details that are obtained through a multi-resolution decomposition of the PAN image into the resampled MS bands [9-12]. Since most of the directional and structural information is contained in the PAN image, several researchers proposed using wavelets [8], contourlets transforms and Laplacian pyramids [9] to extract the spatial details. Currently, some latest MRA algorithms designed for capturing the directional information also have been applied into pansharpening, such as the nonsubsampled contourlet transform [10] and the curvelet transform [11].

Another important category is the model-based fusion approach. On the basis of the study about the image formulation model, some researches regard the solution of the fused image as an inverse optimization problem [12-14]. Sparse representation (SR) has been recently used in signal processing successfully. Combined with SR techniques, researchers obtained some promising fusion results [15-17]. The initial work is proposed by $\mathrm{Li}$ and Yang [15]. Then, Guo et al. [16] proposed an online coupled dictionary learning (OCDL) approach for image fusion, in which a superposition strategy is applied to construct the coupled dictionaries. Zhu and Bamler proposed a new pan-sharpening method named Sparse Fusion of Images (SparseFI) [17], which explores the sparse representation of MS image patches in a dictionary trained only from the PAN image.

\subsection{Motivation and Contributions}

A major limitation of the standard sparse representation is that similar data instances do not guarantee to produce similar sparse weights. Considering that only a few atoms that are closely correlated to the input image patches to the representation, it will be more accurate to represent one image patch using a few correlated atoms, leading to a local representation of image patches. Moreover, in [18], Yu et al. theoretically pointed out that under certain assumptions locality is more essential than sparsity. Motivated by the importance of data locality, locality-constrained linear coding (LCC) [19] and Locality-sensitive dictionary learning [20] has been applied into various image processing areas and achieved some outstanding results, such as image classification [20] and face hallucination [21]. Therefore, we design a novel data locality-constrained method based on sparse representation for remote sensing image pansharpening, called Locality-constrained Sparse Representation (LcSR) in 


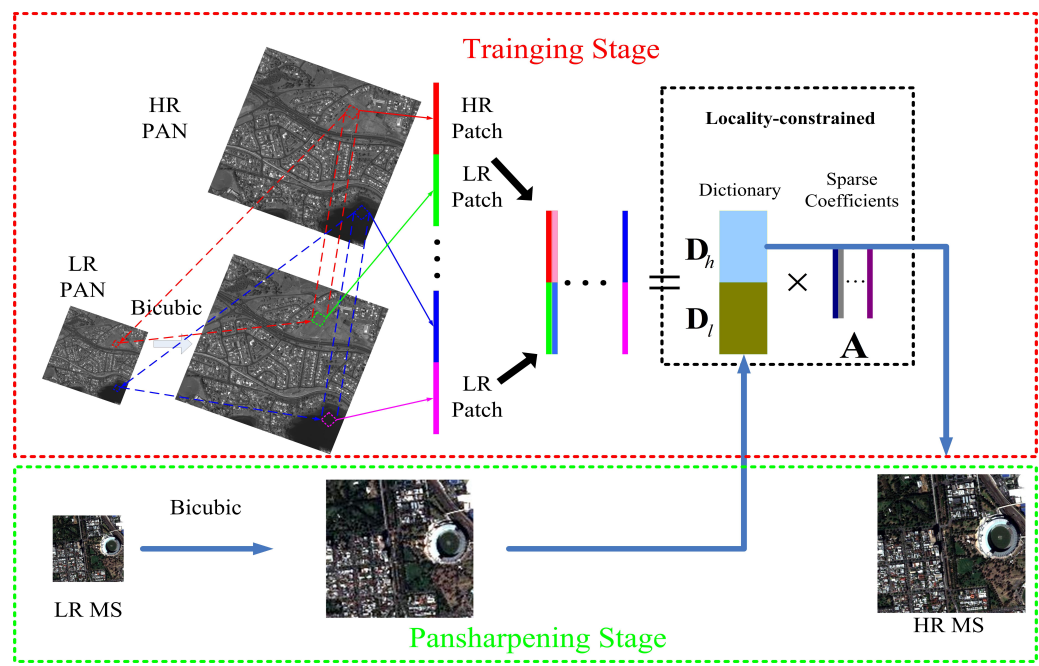

Figure 1: The flowchart of the proposed algorithm.

which a locality constraint is incorporated into the dictionary learning. The proposed method has the following distinct features.

1) The locality constraint captures the fundamental similarities between local patches;

2) The analytical solution is derived for the dictionary update and sparse coding stages with the locality regularization term. Therefore, our learning process is computationally efficient.

3) Joint dictionary learning is proposed, which simultaneously considers the correlation between LR patches as well as between the corresponding HR patches;

The experimental results in this paper indicate that the proposed method can generate competitive fusion results. The flowchart of LcSR method is summarized in Figure 1.

\section{Pansharpening via Locality-constrained Sparse Representation}

In this section, we briefly review the standard sparse representation method in Section 2.1. Section 2.2 presents the Locality-constrained Sparse Representation (LcSR). In Section 2.3, the pansharpening via LcSR is described.

\subsection{Sparse Representation}

In fact, natural images tend to be sparse in an over-complete dictionary, where the image can be represented as a linear combination of only a small number of the dictionary atoms [22]. The sparse representation problem is often represented as:

$$
\underset{\mathbf{D}, \boldsymbol{\alpha}}{\arg \min }\|\mathbf{x}-\mathbf{D} \boldsymbol{\alpha}\|_{2}^{2}+\lambda\|\boldsymbol{\alpha}\|_{0}
$$

where $\mathbf{x}$ is a column vector representing a signal or a lexicographically ordered image patch. $\mathbf{D}=\left[\mathbf{d}_{1}, \mathbf{d}_{2}, \ldots, \mathbf{d}_{K}\right]$ is a matrix representing the dictionary, with each column called as an 
atom. $\boldsymbol{\alpha}$ is a set of the sparse coefficients for $\mathbf{x}$, with most of the coefficients being close to or zero. $\lambda$ is the regularization parameter. $\|\cdot\|_{0}$ denotes the $\ell_{0}$-norm. Since (1) is well known as an NP-hard problem, greedy algorithms such as orthogonal matching pursuit (OMP) are often used to tackle this problem. An alternative approach is to relax (1) to an $\ell_{1}$-norm convex optimization problem:

$$
\underset{\mathbf{D}, \boldsymbol{\alpha}}{\arg \min }\|\mathbf{x}-\mathbf{D} \boldsymbol{\alpha}\|_{2}^{2}+\lambda\|\boldsymbol{\alpha}\|_{1}
$$

where $\|\cdot\|_{1}$ indicates the $\ell_{1}$-norm. Many $\ell_{1}$-norm optimization methods have been proposed to solve this convex problem [23]. Due to the good performance and stability, the least angle regression stagewise (LARS) algorithm [24] has been widely used.

\subsection{Locality-constrained Sparse Representation}

Data locality has been widely utilized in many pattern recognition problems such as dimension reduction [24] and classification [18-20]. Additionally, Local Coordinate Coding (LCC) [19] suggested that locality is more essential than sparsity. Locality must lead to sparsity but not vice versa. This motivates us to propose a Locality-constrained Sparse Representation (LcSR) algorithm for Pansharpening, which is enforced a local constraint term instead of the $\ell_{0}$ or $\ell_{1}$-norm constraint in Eq. (2). The model is formulated as follows:

$$
\underset{\mathbf{D}, \boldsymbol{\alpha}_{i}}{\arg \min }\left\|\mathbf{x}_{i}-\mathbf{D} \boldsymbol{\alpha}_{i}\right\|_{2}^{2}+\lambda\left\|\mathbf{e}_{i} \circ \boldsymbol{\alpha}_{i}\right\|_{2}^{2} \quad \text { s.t. } \sum_{k=1}^{K} \alpha_{i k}=1
$$

where $\mathbf{x}_{i}$ is the $i$-th input image patch, $\boldsymbol{\alpha}_{i} \in R^{K}$ is the $i$-th column of sparse coding matrix $\mathbf{A}=\left[\boldsymbol{\alpha}_{1}, \boldsymbol{\alpha}_{2}, \ldots, \boldsymbol{\alpha}_{N}\right] \in R^{K \times N}, \alpha_{i k}$ is the $k$-th element of $\boldsymbol{\alpha}_{i}$. $\circ$ denotes the element-wise multiplication, and $\mathbf{e}_{i} \in R^{K}$ is the locality adaptor whose $k$-th element is given by $e_{i k}=$ $\left\|\mathbf{y}_{i}-\mathbf{d}_{k}\right\|_{2}^{2}$.

\subsection{Pansharpening via LcSR}

Let $\mathbf{X}_{b}^{l}$ and $\mathbf{X}_{b}^{h}(b=1, \ldots, B)$ denote the $b$ th band of the LR MS image and the HR fused MS image, respectively, where $B$ stands for the number of bands in the MS image. The HR PAN image with a resolution 4 times higher than the HR MS image is represented as $\mathbf{Y}^{h}$ and the LR PAN image is $\mathbf{Y}^{l}$. Our goal is to learn dictionaries for high resolution and low resolution Images. The individual locality-constrained sparse coding problems in the high-resolution and low-resolution image spaces are

$$
\left\{\mathbf{D}_{h}, \boldsymbol{\alpha}_{i}\right\}=\underset{\mathbf{D}_{h}, \boldsymbol{\alpha}_{i}}{\arg \min }\left\|\mathbf{y}_{i}^{h}-\mathbf{D}_{h} \boldsymbol{\alpha}_{i}\right\|_{2}^{2}+\lambda\left\|\mathbf{e}_{i} \circ \boldsymbol{\alpha}_{i}\right\|_{2}^{2} \quad \text { s.t. } \sum_{k=1}^{K} \alpha_{i k}=1
$$

and

$$
\left\{\mathbf{D}_{l}, \boldsymbol{\alpha}_{i}\right\}=\underset{\mathbf{D}_{l}, \boldsymbol{\alpha}_{i}}{\arg \min }\left\|\mathbf{y}_{i}^{l}-\mathbf{D}_{l} \boldsymbol{\alpha}_{i}\right\|_{2}^{2}+\lambda\left\|\mathbf{e}_{i} \circ \boldsymbol{\alpha}_{i}\right\|_{2}^{2} \quad \text { s.t. } \sum_{k=1}^{K} \alpha_{i k}=1
$$

where $\mathbf{y}_{i}^{h}$ and $\mathbf{y}_{i}^{l}$ are the $i$-th extracted patch from $\mathbf{Y}^{h}$ and $\mathbf{Y}^{l}$, respectively. $\mathbf{D}_{h}$ and $\mathbf{D}_{l}$ is a pair dictionary. Forcing the high resolution and low-resolution representations to share the 
same codes, we combine these objectives and obtain

$$
\underset{\mathbf{D}_{h}, \mathbf{D}_{l}, \boldsymbol{\alpha}_{i}}{\arg \min }\left\|\mathbf{y}_{i}^{h}-\mathbf{D}_{h} \boldsymbol{\alpha}_{i}\right\|_{2}^{2}+\left\|\mathbf{y}_{i}^{l}-\mathbf{D}_{l} \boldsymbol{\alpha}_{i}\right\|_{2}^{2}+\lambda\left\|\mathbf{e}_{i} \circ \boldsymbol{\alpha}_{i}\right\|_{2}^{2} \quad \text { s.t. } \sum_{k=1}^{K} \alpha_{i k}=1
$$

Let $\mathbf{y}_{i}^{c}=\left[\begin{array}{c}\mathbf{y}_{i}^{h} \\ \mathbf{y}_{i}^{l}\end{array}\right], \mathbf{D}_{c}=\left[\begin{array}{c}\mathbf{D}_{h} \\ \mathbf{D}_{l}\end{array}\right],(6)$ can be rewritten as

$$
\underset{\mathbf{D}_{h}, \mathbf{D}_{l}, \boldsymbol{\alpha}_{i}}{\arg \min }\left\|\mathbf{y}_{i}^{c}-\mathbf{D}_{c} \boldsymbol{\alpha}_{i}\right\|_{2}^{2}+\lambda\left\|\mathbf{e}_{i} \circ \boldsymbol{\alpha}_{i}\right\|_{2}^{2} \quad \text { s.t. } \sum_{k=1}^{K} \alpha_{i k}=1
$$

Thus we can apply an iterative procedure to update the dictionary $\mathbf{D}_{c}$ and the encoded sparse vector $\boldsymbol{\alpha}_{i}$ in the single dictionary case for our pansharpening purpose. The optimization performs in an alternative scheme over two stages. We call them "sparse coefficients update" and "dictionary update", respectively. In other words, the Eq. (7) is solved by translating to two sub-problems.

In the "sparse coefficients update" stage, fixing the dictionary $\mathbf{D}_{c}=\left[\mathbf{d}_{1}, \mathbf{d}_{2}, \ldots, \mathbf{d}_{K}\right], \boldsymbol{\alpha}_{i}$ will be the solution of the following optimization problem

$$
\underset{\boldsymbol{\alpha}_{i}}{\arg \min }\left\|\mathbf{y}_{i}^{c}-\mathbf{D}_{c} \boldsymbol{\alpha}_{i}\right\|_{2}^{2}+\lambda\left\|\mathbf{e}_{i} \circ \boldsymbol{\alpha}_{i}\right\|_{2}^{2} \quad \text { s.t. } \sum_{k=1}^{K} \alpha_{i k}=1
$$

where $\mathbf{y}_{i}^{c}$ is an extracted patch from $\mathbf{Y}_{c}$. To optimize the objective function (8), we consider be the Lagrange function $L\left(\boldsymbol{\alpha}_{i}, \boldsymbol{\beta}\right)$, which is defined as

$$
L\left(\boldsymbol{\alpha}_{i}, \beta\right)=\left\|\mathbf{y}_{i}^{c}-\mathbf{D}_{c} \boldsymbol{\alpha}_{i}\right\|_{2}^{2}+\lambda\left\|\mathbf{e}_{i} \circ \boldsymbol{\alpha}_{i}\right\|_{2}^{2}+\beta\left(\mathbf{1}^{T} \boldsymbol{\alpha}_{i}-1\right)
$$

where $\mathbf{1}$ is a $K \times 1$ column vector of ones. Let us define a covariance matrix $\mathbf{C}$ for $\mathbf{y}_{i}^{c}$ as $\mathbf{C}=\left(\mathbf{y}_{i}^{c} \mathbf{1}^{T}-\mathbf{D}_{c}\right)^{T}\left(\mathbf{y}_{i}^{c} \mathbf{1}^{T}-\mathbf{D}_{c}\right)$, and is a diagonal matrix whose nonzero elements are the entries of $\mathbf{e}_{i}$. Following [20], we can get the analytical solution of (8) as

$$
\alpha_{i}=\left(\mathbf{C}+\lambda \operatorname{diag}\left(\mathbf{e}_{i}\right)^{2}\right)^{-1} \mathbf{1} /\left(\mathbf{1}^{T}\left(\mathbf{C}+\lambda \operatorname{diag}\left(\mathbf{e}_{i}\right)^{2}\right)^{-1} \mathbf{1}\right)
$$

On the other hand, the dictionary update stage needs to solve

$$
\underset{\mathbf{D}_{c}}{\arg \min }\left\|\mathbf{y}_{i}^{c}-\mathbf{D}_{c} \boldsymbol{\alpha}_{i}\right\|_{2}^{2}+\lambda\left\|\mathbf{e}_{i} \circ \boldsymbol{\alpha}_{i}\right\|_{2}^{2}
$$

We denote $F\left(\mathbf{D}_{c}\right)=\left\|\mathbf{y}_{i}^{c}-\mathbf{D}_{c} \boldsymbol{\alpha}_{i}\right\|_{2}^{2}+\lambda\left\|\mathbf{e}_{i} \circ \boldsymbol{\alpha}_{i}\right\|_{2}^{2}$ and let its partial derivatives with respect to the atom $\mathbf{d}_{k}$ equal to zero. We have

$$
\frac{\partial F}{\partial \mathbf{d}_{k}}=\sum_{i=1}^{N}-2 \alpha_{i k}\left(\mathbf{y}_{i}^{c}-\mathbf{D}_{c} \boldsymbol{\alpha}_{i}\right)-2 \lambda\left(\alpha_{i k}\right)^{2}\left(\mathbf{y}_{i}^{c}-\mathbf{d}_{k}\right)=0 k \in\{1,2, \ldots, K\}
$$

Let the matrices $\mathbf{U}_{\mathbf{D}_{c}}=\sum_{i=1}^{N}\left(\begin{array}{cccc}(1+\lambda) \alpha_{i 1}^{2} & \alpha_{i 1} \alpha_{i 2} & \cdots & \alpha_{i 1} \alpha_{i K} \\ \alpha_{i 1} \alpha_{i 2} & (1+\lambda) \alpha_{i 2}^{2} & \cdots & \alpha_{i 2} \alpha_{i K} \\ \vdots & \vdots & \ddots & \vdots \\ \alpha_{i 1} \alpha_{i K} & \alpha_{i 2} \alpha_{i K} & \cdots & (1+\lambda) \alpha_{i K}^{2}\end{array}\right)$, 


$$
\mathbf{V}_{\mathbf{D}_{c}}=\sum_{i=1}^{N}\left(\begin{array}{c}
\alpha_{i 1}\left(1+\lambda \alpha_{i 1}\right)\left(\mathbf{y}_{i}^{c}\right)^{T} \\
\alpha_{i 2}\left(1+\lambda \alpha_{i 2}\right)\left(\mathbf{y}_{i}^{c}\right)^{T} \\
\vdots \\
\alpha_{i K}\left(1+\lambda \alpha_{i K}\right)\left(\mathbf{y}_{i}^{c}\right)^{T}
\end{array}\right) \text {. Eq. (12) can be reformulated as } \mathbf{U}_{\mathbf{D}_{c}} \mathbf{D}_{c}^{T}-\mathbf{V}_{\mathbf{D}_{c}}=
$$

0 .Then, it's easy to obtain the solution $\mathbf{D}_{c}=\left(\mathbf{U}_{\mathbf{D}_{c}}{ }^{-1} \mathbf{V}_{\mathbf{D}_{c}}\right)^{T}$.

Overall, in our work, we alternate between the two steps, sparse coding and dictionary updating, for obtaining the optimal solutions $\mathbf{A}$ and $\mathbf{D}_{c}$.

In the pansharpened stage, $\mathbf{x}_{b-m}^{l}$ is an LR multispectral patch, indexed as $m$, in the $b$-th channel. It's sparse coefficients can be calculated by following step:

$$
\underset{\boldsymbol{\alpha}_{b-m}}{\arg \min }\left\|\mathbf{x}_{b-m}^{l}-\mathbf{D}_{l} \alpha_{b-m}\right\|_{F}^{2}+\lambda\left\|\mathbf{e}_{b-m} \circ \boldsymbol{\alpha}_{b-m}\right\|_{2}^{2} \quad \text { s.t. } \sum_{k=1}^{K} \alpha_{b-m, k}=1
$$

The final sharpened multispectral image patches are reconstructed by

$$
\mathbf{x}_{b-m}^{h}=\mathbf{D}_{h} \boldsymbol{\alpha}_{b-m}
$$

At last, we tile all patches in all individual channels to get the desired pansharpened image $\mathbf{X}_{b}^{h}$ $(b=1, \ldots, B)$. For the overlapping areas, an average of all patches at each pixel is computed as the final fused results.

\section{Experimental Results and Analysis}

To assess the quality of the fusion results quantitatively, simulation experiments are performed on two data sets, i.e. QuickBird and WorldView-2. Five typical evaluation metrics were adopted to assess the quality of fused images. The correlation coefficient (CC) and root-mean-square error were calculated for each band between the fused MS images and the reference original MS image. Erreur relative globale adimensionnelle de synthílse (ERGAS) and Q4 , which are two comprehensive evaluation indexes, provide unique measures of the fusion performance for all the MS bands. Furthermore, the spectral angle mapper (SAM) index was also considered to measure the spectral distortion. The ideal values of CC, ERGAS, RASE, SAM and Q4 are 1,0, 0, 0 and 1, respectively.

To validate the effectiveness of the proposed method for pansharpening, we compared with four state-of-the-art fusion algorithms: AIHS (Adaptive IHS) [6], Brovey [25], Wavelet [8] and SparseFI [17]. The implementations of the AIHS, Brovey and Wavelet method are available online. The default parameters given in their implementations are adopted. The LR patch size in the SparseFI method is $7 \times 7$. A total of 10,000 patch pairs are selected to construct the dictionary pairs. As to our LcSR method, the LR patch size is selected $7 \times 7$, the dictionary size is 768 .

\subsection{Effects of Dictionary Size}

In this subsection, we evaluate the effect of dictionary size on pansharpening, i.e., the number of atoms in dictionary. From the sampled image patch pairs, we train four dictionaries of size $256,512,768$, and 1024 , and apply them to the same remote sensing image. The results are evaluated both visually and quantitatively in Figure 2, Figure 3 and Table 1. 


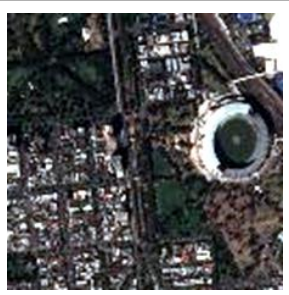

(a)

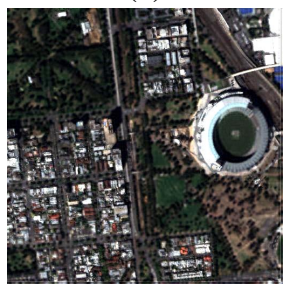

(d)

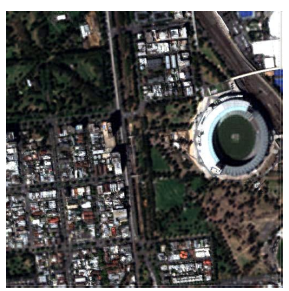

(b)

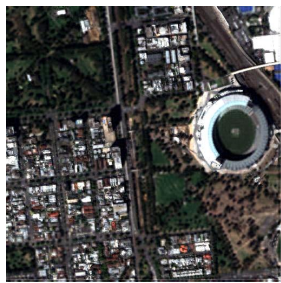

(e)

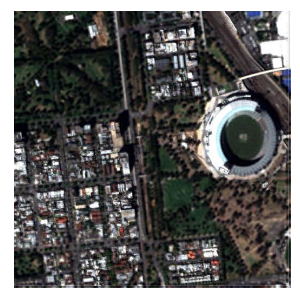

(c)

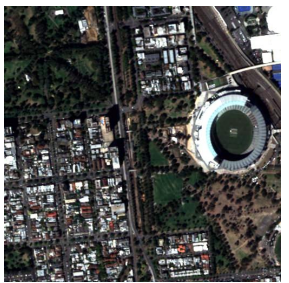

(f)

Figure 2: The visual effects of different dictionary sizes on the pansharpening of WorldView2 image: (a) LR MS image; (b)256; (c) 512; (d) 768;(e) 1024; (f) HR MS image.

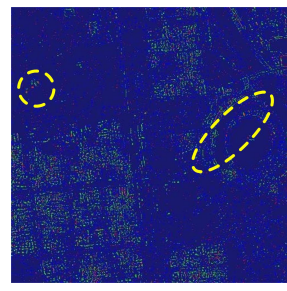

(a)

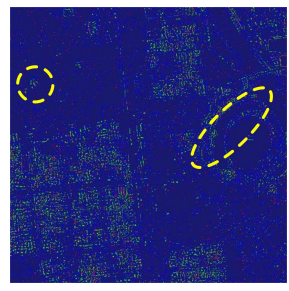

(b)

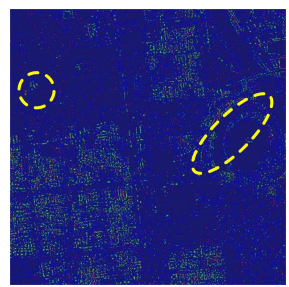

(c)

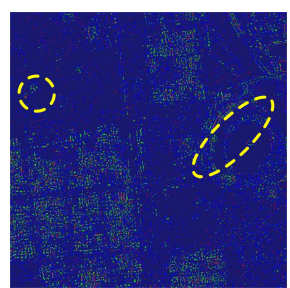

(d)

Figure 3: The difference images of different dictionary sizes on the pansharpening of WorldView-2 image: (a) 256; (b) 512; (c) 768;(d) 1024.

Figure 2 exhibits the fused results for the Worldview-2 image using dictionaries of different sizes. Human visual system is not sensitive to the weak spectral distortions. Always we justify the distortion from the color change [26]. Thus, we display the difference of pixel values measured between each pansharpened image and the reference HR MS image. Deep blue represents the smallest difference, while the red means the largest difference. Figure 3 shows the difference image under different dictionary sizes. We indeed observe the artifacts will gradually diminish with larger dictionaries (i. e. the subtle differences in the yellow circle in Figure 3). In Table 1, we list five indexes of the pansharpened image for dictionaries of different sizes. As shown in the table, the performance of the proposed method can be improved further through increasing the dictionary size, but at higher computational cost. Therefore, one chooses an appropriate dictionary size as a trade-off between pansharpening quality and computation cost. In our experiments, the dictionary size is set 768 .

\subsection{Simulation Results and Analysis}

In the following, the performance of the proposed method is evaluated on a pair of simulated QuickBird images. Figure 4(a) and (b) shows the low-resolution MS image with a resolu- 


\begin{tabular}{|c|c|c|c|c|c|}
\hline dictionary size & CC & RMSE & SAM & ERGAS & Q4 \\
\hline \hline 256 & 0.9806 & 0.0508 & 3.1367 & 3.6174 & 0.7492 \\
512 & 0.9818 & 0.0487 & 3.1208 & 3.5622 & 0.7513 \\
768 & 0.9824 & 0.0479 & 3.1183 & 3.4994 & 0.7521 \\
1024 & 0.9830 & 0.0462 & 3.1125 & 3.4237 & 0.7536 \\
\hline
\end{tabular}

Table 1: The objective indexes of the pansharpened images with dictionaries of different sizes.

\begin{tabular}{|c|c|c|c|c|c|}
\hline Method & CC & RMSE & SAM & ERGAS & Q4 \\
\hline \hline AIHS & 0.9442 & 0.909 & 5.5804 & 5.8664 & 0.7521 \\
Brovey & 0.9330 & 0.1201 & 6.6128 & 7.2698 & 0.7189 \\
Wavelet & 0.9616 & 0.1309 & 6.6189 & 8.3254 & 0.6661 \\
SparseFI & 0.9641 & 0.0845 & 4.1082 & 5.5103 & 0.7727 \\
LcSR & $\mathbf{0 . 9 6 7 7}$ & $\mathbf{0 . 0 7 9 6}$ & $\mathbf{4 . 0 0 0 3}$ & $\mathbf{5 . 2 1 1 6}$ & $\mathbf{0 . 7 7 8 5}$ \\
\hline
\end{tabular}

Table 2: Objective performance for the different pansharpening methods on QuickBird data.(The bold black values mrean the best performance)

tion of $11.2 \mathrm{~m}$ and PAN image with a resolution of $2.8 \mathrm{~m}$, respectively. The original MS image at $2.8-\mathrm{m}$ resolution is used as the reference image, as shown in Figure 4(h). There are many varieties of ground objects such as vegetation, buildings and roads. The fused images of various methods are reported in Figure 4(c)-(g). Compared with the reference image [Figure 4(h)], the Brovey method can preserves the spatial information effectively but suffers from some spectral distortion. Figure 4(e) loses the most detailed information provided by the Wavelet method. The AIHS, SparseFI and LcSR method give the good results in preserving high spectral quality and fine spatial structures. Furthermore, to better visualize the differences among these pan-sharpening methods, we show the difference images between the pan-sharpened images and the reference MS image in Figure 5. It can be obviously seen in Figure 5(e) that the proposed method causes the smallest spectral distortion and spatial structural difference for most regions, whereas the other methods all retain more spatial structural information and cause more spectral distortion, as shown in the difference images. Finally, Tables 2 reports the quantitative results of different methods corresponding to the pan-sharpening examples. The best results in the CC, SAM, ERGAS, RMSE and Q4 metrics among all the compared methods, which also indicates that the pan-sharpened image of the proposed method performs both the best spectral and spatial qualities.

\section{Conclusion}

In this paper, based on sparse representation and data locality, we presented a novel remote sensing fusion method with the exploitation of data locality, called Locality-constrained Sparse Representation (LcSR). It imposes the locality regularization term for data reconstruction aiming at obtaining the optimal representation of image patches. In addition, the locality adaptor is also embedded in the dictionary update process. Thus, the learned dictionary is sparse and accurate simultaneously. Experimental results on some public databases have demonstrated the superiority of the proposed method by comparing with various wellknown pansharpening methods. 


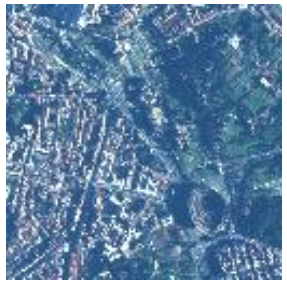

(a)

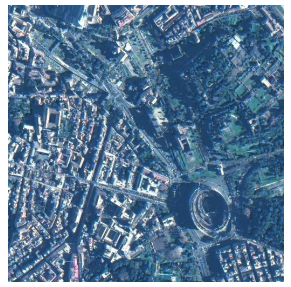

(d)

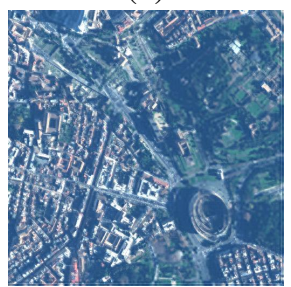

(g)

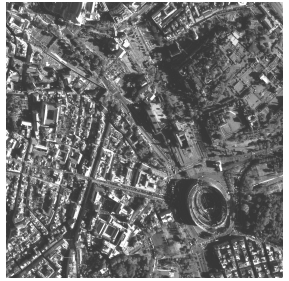

(b)

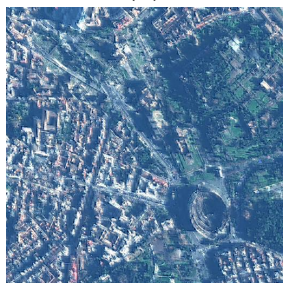

(e)

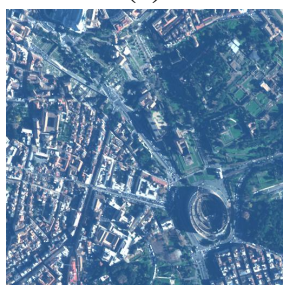

(h)

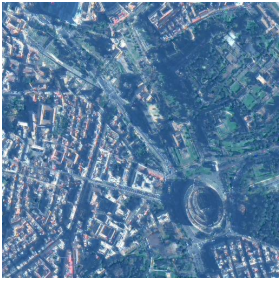

(c)

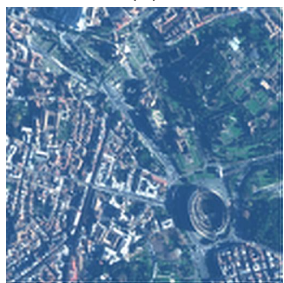

(f)

Figure 4: Source images and pansharpening results obtained by different methods on QuickBird data: (a) LR MS image; (b) PAN image; (c) AIHS; (d) Brovey; (e) Wavelet; (f) SparseFI;(g) LcSR; (h) Reference HR MS image.

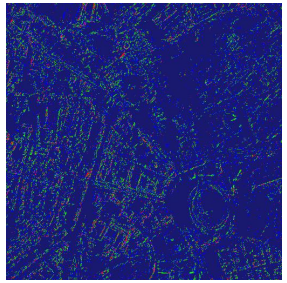

(a)

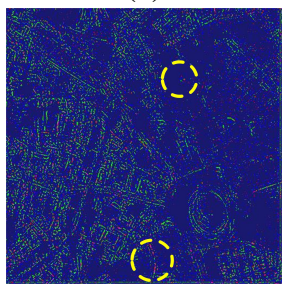

(d)

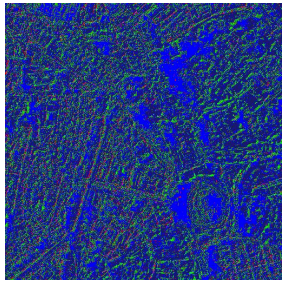

(b)

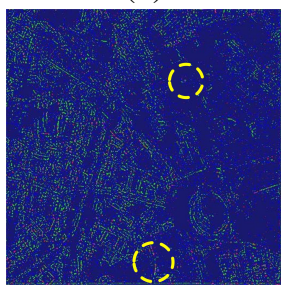

(e)

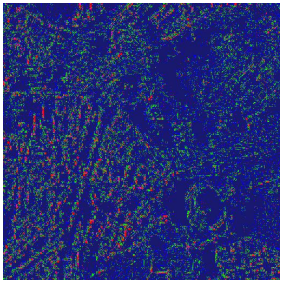

(c)

Figure 5: Difference images (blue means small differences, and red means large differences)between each pansharpened MS images and the reference HR MS image shown in Figure 4: (a) AIHS; (b) Brovey; (c) Wavelet; (d) SparseFI; (e) LcSR. 


\section{References}

[1] T. M. Tu et al.. An adjustable pan-sharpening approach for IKONOS/QuickBird/GeoEye-1/WorldView-2 imagery. IEEE J. Sel. Topics Appl Earth Observ. Remote Sens., 5(1):125-134, 2012.

[2] H. T. Yin and S. T. Li. Pansharpening with multiscale normalized nonlocal means filter: A Two-Step Approach. IEEE Trans. Geosci. Remote Sens., 53(10): 5734-5745, 2015.

[3] S. Z. Tang et al.. Pan-sharpening using 2D CCA. Remote Sens. Lett., 6(5):341-350, 2015.

[4] T.-M. Tu, P. S. Huang, C.-L. Hung, and C.-P. Chang. A fast intensity hue-saturation fusion technique with spectral adjustment for IKONOS imagery. IEEE Geosci. Remote Sens. Lett., 1(4): 309-312, 2004.

[5] P. S. Chavez, S. C. Sides, and J. A. Anderson. Comparison of three different methods to merge multiresolution and multispectral data Landsat TM and SPOT panchromatic. Photogramm. Eng. Remote Sens., 57(3):295-303, 1991.

[6] S. Rahmani et al.. An adaptive IHS pan-sharpening method. IEEE Geosci. Remote Sens. Lett., 7(4):746-750, 2010.

[7] R. Masoudi and P. Kabiri. New intensity-hue-saturation Pan-sharpening method based on texture analysis and genetic algorithm-adaption. J. of Appl. Remote Sens., 8(1):083640, 2014.

[8] X. Otazu, M. Gonzalez-Audicana, O. Fors, and J. Nunez. Introduction of Sensor Spectral Response into Image Fusion Methods. Application to Wavelet-Based Methods. IEEE Trans. Geosci. Remote Sens., 43(10): 2376-2385, 2005.

[9] B. Aiazzi, L. Alparone, S. Baronti, A. Garzelli, and M. Selva. MTF tailored multiscale fusion of high-resolution MS and pan imagery. Photogram. Eng. Remote Sens., 72(5): 591-596, 2006.

[10] A. G. Mahyari and M. Yazdi. Panchromatic and multispectral image fusion based on maximization of both spectral and spatial similarities. IEEE Trans. Geosci. Remote Sens., 49(6): 1976-1985, 2011.

[11] F. Nencini, A. Garzelli, S. Baronti, and L. Alparone. Remote sensing image fusion using the curvelet transform. Inf. Fusion, 8(2): 143-156, 2007.

[12] H. Shen, X. Meng, and L. Zhang. An integrated framework for the spatiotemporal-spectral fusion of remote sensing images. IEEE Trans. Geosci. Remote Sens., 54(12):7135-7148, 2016.

[13] R. Molina et al.. Variational posterior distribution approximation bayesian super resolution reconstruction of multispectral images. Appl. Comput. Harmon. Anal., special issue on “Mathematical Imaging," Part II, 24(2):251-267, 2008.

[14] P. Liu, L. Xiao, J. Zhang, B. Naz. Spatial-hessian-feature-guided variational model for pan-sharpening. IEEE Trans. Geosci. Remote Sens.. 54(4): 2235-2253, 2016. 
[15] S. Li, and B. Yang. A new pan-sharpening method using a compressed sensing technique. IEEE Trans. Geosci. Remote Sens., 49(2):738-746, 2011.

[16] M. Guo , H. Zhang , J. Li , et al.. An online coupled dictionary learning approach for remote sensing image fusion. IEEE Journal of Selected Topics in Applied Earth Observations and Remote Sensing, 7(4): 1284-1294, 2014.

[17] X. Zhu, and R. Bamler. A sparse image fusion algorithm with application to Pansharpening. IEEE Trans. Geosci. Remote Sens., 51(5): 2827-2836, 2013.

[18] K. Yu, T. Zhang, and Y. Gong. Nonlinear learning using local coordinate coding. NIPS, 2009.

[19] J. Wang, J. Yang, K. Yu, et al. Locality-constrained Linear Coding for image classification. Computer Vision and Pattern Recognition, 3360-3367, 2010.

[20] C. P. Wei, Y. W. Chao, Y. R. Yeh, et al.. Locality-sensitive dictionary learning for sparse representation based classification. Pattern Recognition, 46(5): 1277-1287, 2013.

[21] J. Jiang, R. Hu, Z. Wang, et al.. Noise robust face hallucination via locality-constrained representation. IEEE Transactions on Multimedia, 16(5): 1268-1281, 2014.

[22] J. Yang, J. Wright, T. S. Huang, et al.. Image super-resolution via sparse representation. IEEE Transactions on Image Processing, 19(11):2861-2873, 2010.

[23] B. Efron, T. Hastie, I. Johnstone, and R. Tibshirani. Least angle regression. The Annals of statistics, 32(2): 407-499, 2004.

[24] S. T. Roweis, L. K. Saul. Nonlinear dimensionality reduction by locally linear embedding. Science, 290 (5500): 2323-2326, 2000.

[25] Y. Zhang. Problems in the Fusion of Commercial High-Resolution Satellite Images as Well as LANDSAT 7 Images and Initial Solutions. Proceedings of the GeoSpatial Theory, Processing, and Applications, 34(4): 9-12, 2002.

[26] X. Kang, S. Li, and J. A. Benediktsson. Pansharpening With Matting Model. IEEE Trans. Geosci. Remote Sens., 52(8): 5088-5099, 2014. 